and class I molecules (19-21), in particular those associated with T1D susceptibility, is increasing. This offers researchers the opportunity to test such binding predictions and may provide an in vivo readout of the responses induced by a given peptide also in relation to dose and route of administration. Screening of candidate peptides for clinical trials using such humanized mouse models could guide the choice of peptides for future prevention trials and might maximize our chances of achieving both efficacy and safety.

1. Nepom, G.T. 2002. Therapy of autoimmune diseases: clinical trials and new biologics. Curr. Opin. Immunol. 14:812-815.

2. Liu, E., and Eisenbarth, G.S. 2002. Type 1A diabetes mellitus-associated autoimmunity. Endocrinol. Metab. Clin. North Am. 31:391-410.

3. Daniel, D., and Wegmann, D.R. 1996. Protection of nonobese diabetic mice from diabetes by intranasal or subcutaneous administration of insulin peptide B-(9-23). Proc. Natl. Acad. Sci. U. S. A. 93:956-960.

4. Wong, F.S., et al. 1999. Identification of an MHC class I-restricted autoantigen in type 1 diabetes by screening an organ-specific cDNA library. Nat. Med. 5:1026-1031.

5. Chen, W., et al. 2001. Evidence that a peptide span- ning the B-C junction of proinsulin is an early autoantigen epitope in the pathogenesis of type 1 diabetes. J. Immunol. 167:4926-4935.

6. Rudy, G., et al. 1995. Similar peptides from two beta cell autoantigens, proinsulin and glutamic acid decarboxylase, stimulate T cells of individuals at risk for insulin-dependent diabetes. Mol. Med. 1:625-633

7. Muir, A., Schatz, D., and Maclaren, N. 1993. Antigen-specific immunotherapy: oral tolerance and subcutaneous immunization in the treatment of insulin-dependent diabetes. Diabetes Metab. Rev 9:279-287.

8. Bergerot, I., Fabien, N., Maguer, V., and Thivolet, C 1994. Oral administration of human insulin to NOD mice generates CD4+ T cells that suppress adoptive transfer of diabetes. J. Autoimmun. 7:655-663.

9. Maron, R., Guerau-de-Arellano, M., Zhang, X., and Weiner, H.L. 2001. Oral administration of insulin to neonates suppresses spontaneous and cyclophosphamide induced diabetes in the NOD mouse. J. Autoimmun. 16:21-28.

10. Atkinson, M., Maclaren, N., Luchetta, R., and Burr, I. 1990. Insulitis and diabetes in NOD mice reduced by prophylactic insulin therapy. Diabetes. 39:933-937.

11. Harrison, L.C., Dempsey-Collier, M., Kramer, D.R., and Takahashi, K. 1996. Aerosol insulin induces regulatory CD8 gamma delta $\mathrm{T}$ cells that prevent murine insulin-dependent diabetes. J. Exp. Med. 184:2167-2174.

12. Diabetes Prevention Trial-Type 1 Diabetes Study Group. 2002. Effects of insulin in relatives of patients with type 1 diabetes mellitus. N. Engl. J. Med. 346:1685-1691.
13. Harrison, L.C., et al. 1999. Intranasal insulin trial (INIT) in preclinical type 1 diabetes. Diabetes. 48:A206. (Abstr.)

14. Tian, J., Olcott, A.P., and Kaufman, D.L. 2002 Antigen-based immunotherapy drives the precocious development of autoimmunity. J. Immunol. 169:6564-6569.

15. Cetkovic-Cvrlje, M., et al. 1997. Retardation or acceleration of diabetes in NOD/Lt mice mediated by intrathymic administration of candidate beta-cell antigens. Diabetes. 46:1975-1982.

16. Liu, E., et al. 2002. Anti-peptide autoantibodies and fatal anaphylaxis in NOD mice in response to insulin self-peptides B:9-23 and B:13-23. J. Clin. Invest. 110:1021-1027. doi:10.1172/JCI200215488.

17. Pedotti, R, et al. 2003. Severe anaphylactic reactions to glutamic acid decarboxylase (GAD) self peptides in NOD mice that spontaneously develop autoimmune type 1 diabetes mellitus. BMC Immunol. 4:2

18. Martinez, N.R., et al. 2003. Disabling an integral CTL epitope allows suppression of autoimmune diabetes by intranasal proinsulin peptide. J. Clin. Invest. 111:1365-1371. doi:10.1172/JCI200317166.

19. Das, P., Abraham, R., and David, C. 2000. HLA transgenic mice as models of human autoimmune diseases. Rev. Immunogenet. 2:105-114.

20. Wong, F.S., Moustakas, A.K., Wen, L., Papadopoulos, G.K., and Janeway, C.A., Jr. 2002. Analysis of structure and function relationships of an autoantigenic peptide of insulin bound to $\mathrm{H}-2 \mathrm{~K}(\mathrm{~d})$ that stimulates CD8 $\mathrm{T}$ cells in insulin-dependent diabetes mellitus. Proc. Natl. Acad. Sci. U. S. A 99:5551-5556.

21. Wong, F.S., and Wen, L. 2003. The study of HLA class II and autoimmune diabetes. Curr. Mol. Med. 3:1-15.

\title{
Tissue glycogen content and glucose intolerance
}

\section{Masato Kasuga, Wataru Ogawa, and Takeshi Ohara}

Division of Diabetes and Digestive and Kidney Diseases, Department of Clinical Molecular Medicine, Kobe University Graduate School of Medicine, Kobe, Japan

J. Clin. Invest. 111:1282-1284 (2003). doi:10.1172/JCI200318526.

Insulin stimulates glycogen synthesis in the the liver and skeletal muscle. After a mixed meal, the secretion of insulin from pancreatic $\beta$ cells thus results in

Address correspondence to: Masato Kasuga, Division of Diabetes and Digestive and Kidney Diseases, Department of Clinical Molecular Medicine, Kobe University Graduate School of Medicine,

5-1 Kusunoki-cho 7-chome, Chuo-ku, Kobe 650-0017, Japan. Phone: 81-78-382-5860;

Fax: 81-78-382-2084;

E-mail: kasuga@med.kobe-u.ac.jp.

Conflict of interest: The authors have declared that no conflict of interest exists.

Nonstandard abbreviations used: glycogen synthase kinase-3 (GSK-3); protein phosphatase 1 (PP1); protein targeting to glycogen (PTG). about $20 \%$ and $30 \%$ of the carbohydrate intake being stored in the form of glycogen in the liver and skeletal muscle, respectively $(1,2)$. Defects in this process can therefore be a major contributor to postprandial hyperglycemia. Indeed, the glycogen contents of the liver and skeletal muscle are reduced in individuals with type 2 diabetes $(3,4)$.

Glycogen metabolism is controlled predominantly by the coordinated action of two enzymes, glycogen synthase and glycogen phosphorylase, both of which are regulated by phosphorylation and allosteric modulators. Insulin promotes the net dephosphorylation of both glycogen synthase and glycogen phosphorylase through the inhibition of protein kinases and the activation of protein phosphatases. Among the protein kinases, glycogen synthase kinase-3 (GSK-3) is thought to be an important target for insulin in its stimulation of glycogen synthase activity $(5,6)$. Among the protein phosphatases, protein phosphatase 1 (PP1) has been implicated in this action of insulin (6).

PP1 is an abundant protein serinethreonine phosphatase that is expressed in all compartments of eukaryotic cells. The catalytic subunit of PP1 thus interacts with a wide variety of targeting subunits that localize it to specific sites within the cell. A family of proteins that target PP1 to glycogen and thereby regulate its activity has been identified. These proteins include $\mathrm{G}_{\mathrm{M}}$ (PPP1R3), G (PPP1R4), PTG (protein targeting to glycogen or PPP1R5), and PPP1R6.

\section{Deletion of glycogen-targeting subunits of PP1 (PTG and $G_{M}$ ) in mice}

PTG was cloned as a binding protein of the catalytic subunit of PP1 by Saltiel and coworkers in 1997 (7). This protein 
localizes PP1 to glycogen and also binds to glycogen synthase, glycogen phosphorylase, and phosphorylase kinase within cells. Overexpression of PTG resulted in a marked increase in both basal and insulin-stimulated glycogen synthesis in Chinese hamster ovary cells expressing insulin receptors. In this issue of the JCI, Saltiel and colleagues now describe the generation of mice in which the PTG gene has been deleted (8). Whereas homozygous deletion of the PTG gene resulted in embryonic death, mice missing only one copy of the gene were viable but found to have a reduced glycogen content in adipose tissue, the liver, the heart, and skeletal muscle. Although young (one to two months of age) PTG hemizygous mice exhibited normal glucose tolerance, the animals developed glucose intolerance and insulin resistance (three to four months) and a consequent increase in muscle triglyceride content (18 months) with age.

$\mathrm{G}_{\mathrm{M}}$, also known as PPP1R3 or $\mathrm{R}_{\mathrm{GL}}$, was the first glycogen-binding subunit of PP1 identified and is expressed exclusively in skeletal and cardiac muscle. Homozygous deletion of the $G_{M}$ gene was shown not to result in any obvious defect (9). The homozygous mutant animals manifested normal glucose tolerance and insulin sensitivity at 12 to 24 weeks of age and a weight gain similar to that of their wild-type littermates at up to 12 months of age. Furthermore, these mice exhibited a glucose tolerance similar to that of wild-type animals even after 20 weeks of feeding with a high-fat diet. More recently, however, another $G_{M}$ null mouse line was generated, and the homozygous mutants developed obesity, impaired glucose tolerance, and insulin resistance with age (after 44 to 52 weeks) (10).

Given that the nonfasting glycogen stores of PTG hemizygous mice were reduced by about $50 \%$ in adipose tissue, the liver, and the heart and by about $25 \%$ in skeletal muscle (white fibers), and that both types of $G_{M}$ null mice manifested an approximately $90 \%$ reduction in the glycogen content of skeletal muscle, both of these glycogen-targeting subunits of PP1 (PTG and $\mathrm{G}_{\mathrm{M}}$ ) appear to play an important role in glycogen synthesis in vivo. There are, however, interesting differences between mice deficient in PTG and those lacking $\mathrm{G}_{\mathrm{M}}$. The most obvious such difference is the failure of PTG null mice to develop to term. Determination of the precise time and cause of embryonic death in PTG knockout mice will be important, given that this information may reveal a new and unexpected role for PTG in embryogenesis.

\section{Partitioning of fuel substrates between glycogen and lipid}

Another difference between the effects of PTG and $G_{M}$ deficiency relates to glucose tolerance. Although there are some differences in phenotype between the two lines of $\mathrm{G}_{\mathrm{M}}$ null mice $(9,10)$, glucose intolerance was not detected until at least 11 months of age in either model. In contrast, glucose intolerance was detected at three to four months of age and fasting hyperinsulinemia was already apparent at one to two months of age in PTG hemizygous mice. These differences between PTG and $G_{M}$ mutant animals are suggestive of a difference in the fate of ingested glucose that is not utilized for glycogen synthesis. The second line of $\mathrm{G}_{\mathrm{M}}$ null mice, in which glucose intolerance develops at 11 months of age, manifests an increased deposition of fat in the abdomen and at other sites at 12 months of age, suggesting that glucose not converted to glycogen in skeletal muscle accumulates as triglyceride in adipose tissue. The fact that PTG hemizygous mice showed no difference from wild-type littermates in body weight or in the weight of the liver or fat pads suggests that glucose not converted to glycogen in the liver, adipose tissue, the heart, or skeletal muscle does not accumulate as triglyceride in adipose tissue or the liver. The triglyceride content of hepatocytes and myocytes is thought to correlate negatively with insulin sensitivity $(11,12)$. The triglyceride content of muscle, but not that of the liver, was significantly increased $(+130 \%)$ at 18 months of age in PTG hemizygous mice.

Comparison of the tissues in which glycogen stores are reduced between PTG and $G_{M}$ mutant mice suggests that the deficiency of glycogen in the liver may be responsible for the more profound glucose intolerance of the PTG hemizygous animals. The effect on glucose tolerance of restoring the normal level of PTG gene expression specifically in the liver of the PTG hemizygous mice with the use of an adenovirus vector should help to verify this hypothesis.

Furthermore, PTG hemizygous mice will likely prove to be a good model with which to examine the relation between triglyceride accumulation in hepatocytes or myocytes and insulin resistance. It has not yet been established whether triglyceride accumulation in these cells is the cause or the result of insulin resistance $(13,14)$. Given that fasting hyperinsulinemia was already apparent at one to two months of age in the PTG hemizygotes, it will be interesting to examine both the triglyceride content and insulin signaling in muscle and the liver in animals at this age and at ages up to 18 months. The fact that these mice showed no change in the serum concentration of nonesterified free fatty acids but did exhibit a reduced glucose uptake in white fibers of muscle (at three to four months of age) suggests that they might also provide a unique tool with which to determine the mechanism of triglyceride accumulation in muscle associated with insulin resistance.

\section{Glycogen metabolism and physiology: mouse-human differences}

Finally, we have to consider the possibility that mice may not be an accurate model of human metabolism and physiology. This may be the case especially with regard to glycogen metabolism, given that the patterns of glycogen storage differ between the two species. Thus, although hepatic glycogen content is similar in humans and mice, the glycogen content of mouse muscle is only about $10 \%$ of that of human muscle, when expressed as a percentage of total body glycogen (15). The idea that a reduction in glycogen content of the liver might have a greater effect on glucose tolerance than does a decrease in the glycogen content of muscle, based on observations of mice, should therefore not automatically be assumed to apply to humans. 
The phenotype of mice deficient in PTG suggests that the PTG gene is a candidate gene for type 2 diabetes and insulin resistance in humans. However, previous studies have indicated that PTG gene polymorphism does not contribute to insulin resistance or glucose intolerance $(16,17)$. Given that $\mathrm{G}_{\mathrm{M}}$ gene polymorphism has been associated with insulin resistance in some human populations (18-20), it will be important to reexamine the possible relation between the PTG gene and insulin resistance in humans.

1. Taylor, R., et al. 1996. Direct assessment of liver glycogen storage by ${ }^{13} \mathrm{C}$ nuclear magnetic resonance spectroscopy and regulation of glucose homeostasis after a mixed meal in normal subjects. J. Clin. Invest. 97:126-132.

2. Taylor, R., Price, T.B., Katz, L.D., Shulman, R.G., and Shulman, G.I. 1993. Direct measurement of change in muscle glycogen concentration after a mixed meal in normal subjects. Am. J. Physiol. 265:E224-E229.

3. Magnusson, I., Rothman, D.L., Katz, L.D., Shulman, R.G., and Shulman, G.I. 1992. Increased rate of gluconeogenesis in type II diabetes mellitus. A ${ }^{13} \mathrm{C}$ nuclear magnetic resonance study. J. Clin. Invest. 90:1323-1327.

4. Shulman, G.I., et al. 1990. Quantitation of muscle glycogen synthesis in normal subjects and subjects with non-insulin-dependent diabetes by ${ }^{13} \mathrm{C}$ nuclear magnetic resonance spectroscopy. N. Engl. J. Med. 322:223-228.

5. Cross, D.A.E., Alessi, D.R., Cohen, P., Andjelkovich, M., and Hemmings, B.A. 1995. Inhibition of glycogen synthase kinase- 3 by insulin mediated by protein kinase B. Nature. 378:785-789.

6. Lawrence, J.C., and Roach, P.J. 1997. New insights into the role and mechanism of glycogen synthase activation by insulin. Diabetes. 46:541-547.

7. Printen, J.A., Brady, M.J., and Saltiel, A.R. 1997. PTG, a protein phosphatase 1-binding protein with a role in glycogen metabolism. Science. 275:1475-1478.

8. Crosson, S.M., Khan, A., Printen, J., Pessin, J.E., and Saltiel, A.R. 2003. PTG gene deletion causes impaired glycogen synthesis and developmental insulin resistance. J. Clin. Invest. 111:1423-1432. doi:10.1172/JCI200317975.

9. Suzuki, Y., et al. 2001. Insulin control of glycogen metabolism in knockout mice lacking the muscle-specific protein phosphatase PP1G/ $\mathrm{R}_{\mathrm{GL}}$. Mol. Cell. Biol. 21:2683-2694.

10. Delibegovic, M., et al. 2003. Disruption of the striated muscle glycogen targeting subunit PPP1R3A of protein phosphatase 1 leads to increased weight gain, fat deposition, and development of insulin resistance. Diabetes. 52:596-604.

11. Ryysy, L., et al. 2000. Hepatic fat content and insulin action on free fatty acids and glucose metabolism rather than insulin absorption are associated with insulin requirements during insulin therapy in type 2 diabetic patients. Diabetes. 49:749-758.

12. Jacob, S., et al. 1999. Association of increased intramyocellular lipid content with insulin resistance in lean nondiabetic offspring of type 2 diabetic subjects. Diabetes. 48:1113-1119.

13. Kim, J.K., et al. 2001. Tissue-specific overexpres- sion of lipoprotein lipase causes tissue-specific insulin resistance. Proc. Natl. Acad. Sci. U. S. A 98:7522-7527.

14. Godbole, V., and York, D.A. 1978. Lipogenesis in situ in the genetically obese Zucker fatty rat $(\mathrm{fa} / \mathrm{fa})$ : role of hyperphagia and hyperinsulinaemia. Diabetologia. 14:191-197.

15. Hribal, M.L., Oriente, F., and Accili, D. 2002 Mouse models of insulin resistance. Am. J. Physiol. Endocrinol. Metab. 282:E977-E981.

16. Permana, P.A., Luczy-Bachman, G., and Bogardus, C. 1999. Protein targeting to glycogen/PPP1R5: screening of coding and flanking genomic regions for polymorphisms and association analysis with insulin action in Pima Indians. Biochem. Biophys. Res. Commun. 258:184-186.

17. Hansen, L., et al. 1999. Mutational analysis of the coding regions of the genes encoding protein kinase $B-\alpha$ and $-\beta$, phosphoinositide-dependent protein kinase-1, phosphatase targeting to glycogen, protein phosphatase inhibitor-1, and glycogenin - lessons from a search for genetic vari ability of the insulin-stimulated glycogen synthesis pathway of skeletal muscle in NIDDM patients. Diabetes. 48:403-407.

18. Hansen, L., et al. 1995. A widespread amino acid polymorphism at codon 905 of the glycogenassociated regulatory subunit of protein phosphatase- 1 is associated with insulin resistance and hypersecretion of insulin. Hum. Mol. Genet. 4:1313-1320.

19. Xia, J., et al. 1998. A common variant in $P P P 1 R 3$ associated with insulin resistance and type 2 diabetes. Diabetes. 47:1519-1524

20. Maegawa, H., et al. 1999. The 3'-untranslated region polymorphism of the gene for skeletal muscle-specific glycogen-targeting subunit of protein phosphatase 1 in the type 2 diabetic Japanese population. Diabetes. 48:1469-1472.

\section{Connecting the dots from Toll-like receptors to innate immune cells and inflammatory bowel disease}

\section{David L. Boone and Averil Ma}

Department of Medicine, Ben May Institute for Cancer Research, The University of Chicago, Chicago, Illinois, USA

J. Clin. Invest. 111:1284-1286 (2003). doi:10.1172/JCI200318545.

Address correspondence to: Averil Ma, University of Chicago Hospitals MC 6084, Department of Medicine, 5841 S. Maryland Avenue, Chicago, Illinois 60637, USA. Phone: (773) 834-0687; Fax: (773) 702-2281 E-mail: ama@medicine.bsd.uchicago.edu. Conflict of interest: The authors have declared that no conflict of interest exists. Nonstandard abbreviations used: inflammatory bowel disease (IBD); CTL antigen-4 (CTLA-4); suppressor of cytokine signaling (SOCS); Src homology protein-1 (SHP-1); IL-10 receptor (IL-10R); Toll-like receptor (TLR); pathogen-associated molecular product (PAMP).
The etiologies of inflammatory bowel diseases (IBD) are not known but are thought to involve a genetic predisposition toward exaggerated inflammatory responses to enteric flora. Effective treatments for IBD are therefore predicated on the regulation of inflammatory responses in the intestine. Most current therapeutic agents for IBD, including 5-ASA, prednisone, and anti-TNF antibody are directed at the reduction of proinflammatory molecules. Recently, a number of negative regulatory molecules (e.g., IL-10, TGF- $\beta$, CTL antigen-4 [CTLA-4], Fas, suppressor of cytokine signaling [SOCS] proteins, A20, and Src homology protein-1 [SHP-1]), which either bind to effector immune cells and inhibit their activation (e.g., IL-10, TGF- $\beta$, and CTLA-4), induce programmed cell death (e.g., Fas), or regulate intracellular signaling pathways (e.g., SOCS proteins, SHP-1, and A20), have been identified. These negative regulatory molecules may provide novel therapeutic targets for the treatment of IBD.

\section{IL-10, Stat3, and IBD}

Among these negative regulators of inflammation, IL-10 inhibits multiple cell types, including macrophages (1, 2). The physiological importance of IL-10 is highlighted by the spontaneous development of bowel inflammation in IL-10-deficient $\left(I L-10^{-/-}\right)$ mice $(3,4)$. The inflamed mucosa of $I L-10^{-/-}$mice contains elevated num- 